\title{
Precision aging. Human lifespan has intrinsic limits but measurable outcomes
}

\author{
Warren Ladiges ${ }^{\mathrm{a}, *}$ \\ ${ }^{a}$ Department of Comparative Medicine, School of Medicine, University of Washington, Seattle, WA 98195, USA.
}

A recent article by Pyrkov et al. [1] suggests that progressive loss of physical resilience to aging coincides with an absolute limit of human lifespan in the range of 120 to 150 years. Furthermore, the authors conclude that end of life is an intrinsic biological property independent of internal or external stress factors. Their analysis is based on a log-linear mortality estimate of complete blood count variables, such as neutrophil lymphocyte ratio, as single quantitive measures of the aging process correlated with physical activity data from individual tracking devices. These statistical projections are intriguing and potentially impactful but need to be validated in such a way as to provide practical approaches to defining physical resilience. Once this is established, more accurate predictions can be made for the degree of individual resilience with increasing age, and the power to align with appropriate aging intervention strategies.

One area of current biological research on resilience and aging is focused on developing physical stress tests that actually predict resilience to aging in animal models. For example, in older naïve mice, a non-lethal dose of the drug cyclophosphamide triggers a response in the white blood cell population such that the neutrophil lymphocyte ratio predicts more youthful cognition and physical activity in a subset of mice with increasing age [2]. Another example is immune response to a vaccine. Adult mice vaccinated with the clinically-used vaccine Prevenar 13 could be stratified into high antibody responders and low antibody responders and when followed to older ages, showed high performance and low performance, respectively, in cognition and physical assessment activities [3]. Similar observations were made in a wound healing model where adult mice were administered a small skin punch biopsy and measured for how fast the biopsy closed over several weeks. With increasing age, the rate of closure correlated

* Corresponding author: Warren Ladiges

Mailing address: Department of Comparative Medicine, School of Medicine, University of Washington, Seattle, WA 98195, USA.

Email: wladiges@uw.edu

Received: 03 June 2021 / Accepted: 08 June 2021 with physical performance [4].

These preclinical observations speak for the inclusion of physical stress test variables into the organismal projections described by Pyrkov et al. [1]. This would allow for the development of in vitro assessments that would align with physical resilience and the response patterns associated with the administration of specific physical stress. This approach does not contradict the premise of stress as a non-causal element in aging, but merely provides a means of better defining trajectories of physical resilience with lifespan. By definition, it also implies that individuals with continued robust resilience with increasing age would be in relatively good health and maintain functional independence.

On the other hand, the ability to predict a lack of robustness of physical resilience to aging provides a platform for the possibility of extending healthy aging and lifespan

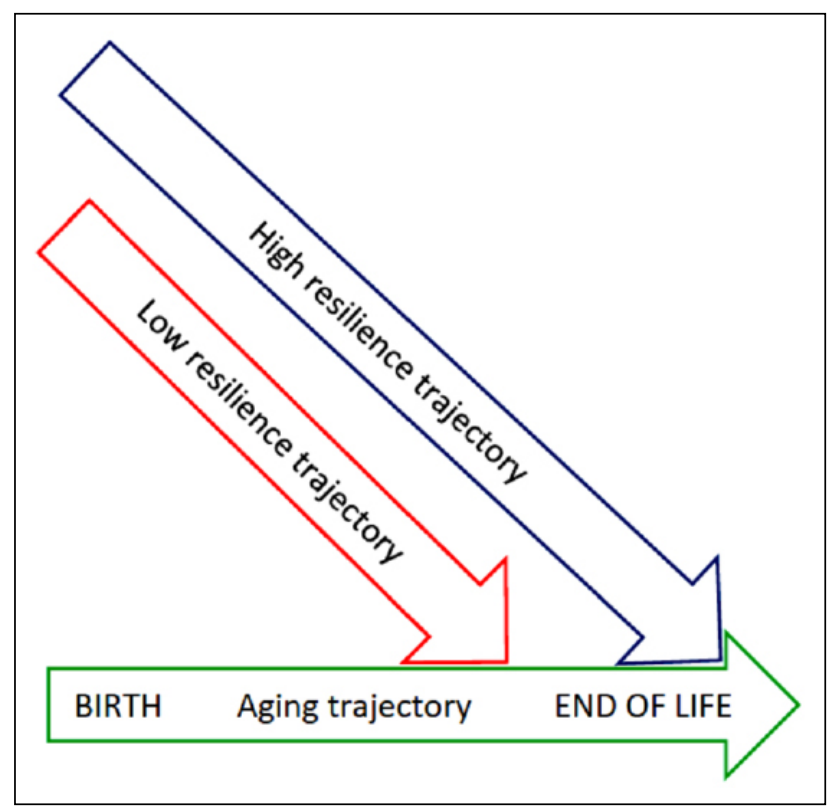

Figure 1. Precision aging and resilience. Precision aging can be used to interrogate resilience trajectories for in vitro stress response patterns on an individual basis. These patterns can then serve as a basis to develop intervention strategies to move resilience in individuals with low resilience trajectories towards a more optimal and projected organismal lifespan endpoint. 
up to the lifespan limits defined by Pyrkov et al. [1]. Aging intervention strategies are being developed that target multiple aging pathways and enhance resilience [5], and could be designed on an individual basis depending on the predictive power of one or several in vitro stress test response patterns. This concept then allows precision medicine to enter into the biology of the aging arena as precision aging (Figure 1), where the objective is not to search for immortality but for ways each individual can maintain a more youthful level of resilience for a healthy and functional life with increasing age but still within an intrinsic lifespan domain.

\section{Declarations}

Financial support and sponsorship: Supported by NIH grants R01 AG057381 (PI, Ladiges).

Conflict of interest: Warren Ladiges is a member of the Editorial Board of Aging Pathobiology and Therapeutics. The author declares no conflict of interest and is not involved in the journal's review or desicions related to this manuscript.

\section{References}

1. Pyrkov T V, Avchaciov K, Tarkhov A E, et al. Longitudinal analysis of blood markers reveals progressive loss of resilience and predicts human lifespan limit. Nature Communications, 2021, 12(1): 1-10.

2. Zhu L, Dou Y, Bjorner M, et al. Development of a cyclophosphamide stress test to predict resilience to aging in mice. GeroScience, 2020, 42(6): 1675-1683.

3. Oveson R, Jiang Z, Izhak M, et al. An immune stress test for resilience to aging: Pneumococcal vaccine response. Aging Pathobiology and Therapeutics, 2020, 2(3): 171172.

4. Jiang Z, Chen J, Wang J, et al. A model for studying cutaneous wound healing and resilience to aging: Ear punch biopsy in old mice. Aging Pathobiology and Therapeutics, 2020, 2(3): 173-175.

5. Ellis M, Ladiges W, Jiang Z. Physical performance is enhanced in old mice fed a short term diet medicated with rapamycin, acarbose, and phenylbutyrate. Aging Pathobiology and Therapeutics, 2021, 3(1): 12-13.

Cite this article as: Warren L. Precision aging. Human lifespan has intrinsic limits but measurable outcomes. Aging Pathobiology and Therapeutics, 2021, 3(2): 39-40. 